\title{
Median nerve entrapment in a callus fracture following a pediatric both-bone forearm fracture: A case report and literature review
}

\author{
Amine Fourati ${ }^{1}$, Iyadh Ghorbel ${ }^{1}$, Amir Karra ${ }^{1}$, Mohamed Habib Elleuch ${ }^{2}$, Khalil Ennouri ${ }^{1}$ \\ Departments of ${ }^{1}$ Plastic Surgery and ${ }^{2}$ Physical Medicine and Rehabilitation, Habib Bourguiba Hospital, Sfax, Tunisia
}

Forearm fractures are common injuries in childhood. Median nerve entrapment is a rare complication of forearm fractures, but several cases have been reported in the literature. This case report discusses the diagnosis and management of median nerve entrapment in a 13-yearold male who presented acutely with a both-bone forearm fracture and numbness in the median nerve distribution. Following the delayed diagnosis, surgical exploration revealed complete nerve entrapment and a nerve graft was performed.

Keywords Humans / Forearm injuries / Fractures, bone / Median nerve / Transplants
Correspondence: Amine Fourati Department of Plastic Surgery, Habib Bourguiba Hospital, Faculty of Medicine of Sfax, Sfax University, Sfax 3000, Tunisia

Tel: $+216-28020002$

Fax: +216-74243427

E-mail: amine_fourati@ medecinesfax.org

Received: 4 Nov 2017 • Revised: 13 Mar 2018 • Accepted: 14 Apr 2018

pISSN: 2234-6163 • elSSN: 2234-6171 • https://doi.org/10.5999/aps.2017.01606• Arch Plast Surg 2019;46:171-175

\section{INTRODUCTION}

Forearm fractures are common injuries in childhood. Fractures of the distal third of the radius and ulna account for $75 \%$ of all pediatric both-bone forearm fractures [1,2]. Median nerve entrapment is a rare complication of forearm fractures, but several cases have been reported in the literature [1-11].

\section{CASE}

A 13-year-old right-hand-dominant boy fell off a bicycle. He was seen at a local emergency room in December 2013. A physical examination revealed a deformed and painful left forearm without neurological or circulatory impairment. Radiographs showed a greenstick both-bone distal fracture (Fig. 1). The patient underwent a closed reduction with satisfactory post-manipulation radiographs.

Weekly examinations showed no fracture displacement and normal bone healing, but the patient complained of numbness in his thumb, index finger, and middle finger. The sensory loss was thought to be caused by neurapraxia, and the patient was advised that the numbness would resolve spontaneously.

Two months later, the plaster was removed, radiographs showed union of both fractured bones with callus bridging (Fig. 2), and the patient noted improved sensibility in the thumb.

Six months after the initial injury, the patient presented to the emergency room after burning his middle finger pulp. On examination, there was no 2-point discrimination in the median nerve distribution of the left hand.

Finally, the patient was referred to us for evaluation. Examination revealed a thenar eminence atrophy, decreased power of the abductor pollicis brevis, and the Tinel sign was positive over the fracture site and the wrist. The flexor digitorum profundus extending into the index finger and the flexor pollicis longus muscles were normal in muscle testing. A neurophysiological study revealed abnormal median nerve conduction distally, and nee- 


\section{Fig. 1. The radiographs of the both-bone forearm fracture}

The radiographs were obtained in the emergency room, with an anteroposterior view $(A)$ and lateral view of the radial and ulnar fracture with dorsal angulation (B).
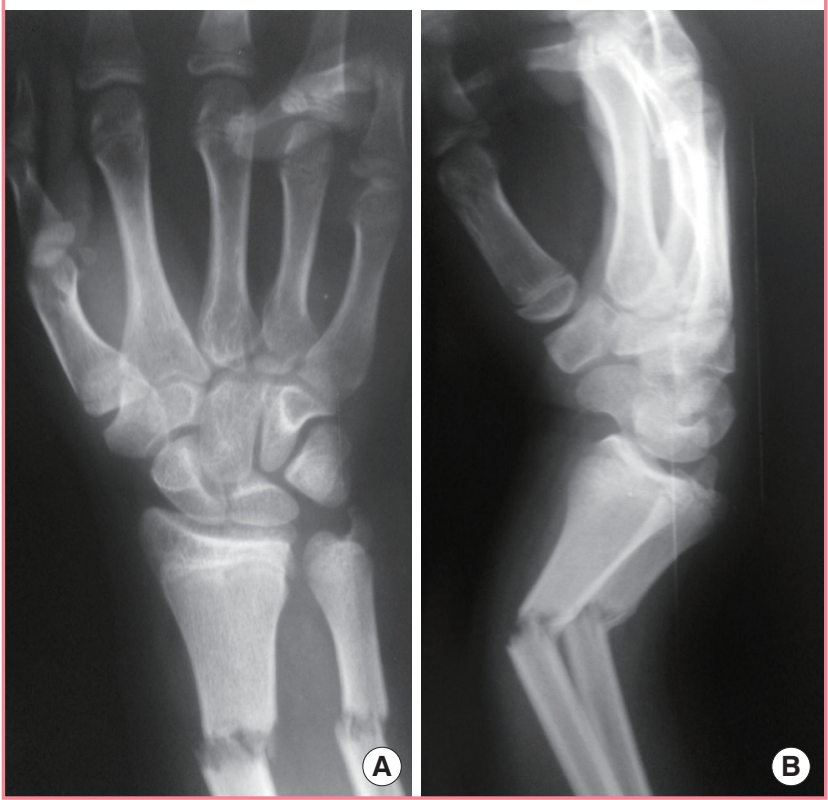

Fig. 2. The radiographic follow-up after 2 months

Radiographs showed the union of the fracture without angulation in the coronal plane $(\mathrm{A})$ and $15^{\circ}$ of tolerated angulation in the sagittal plane (B).
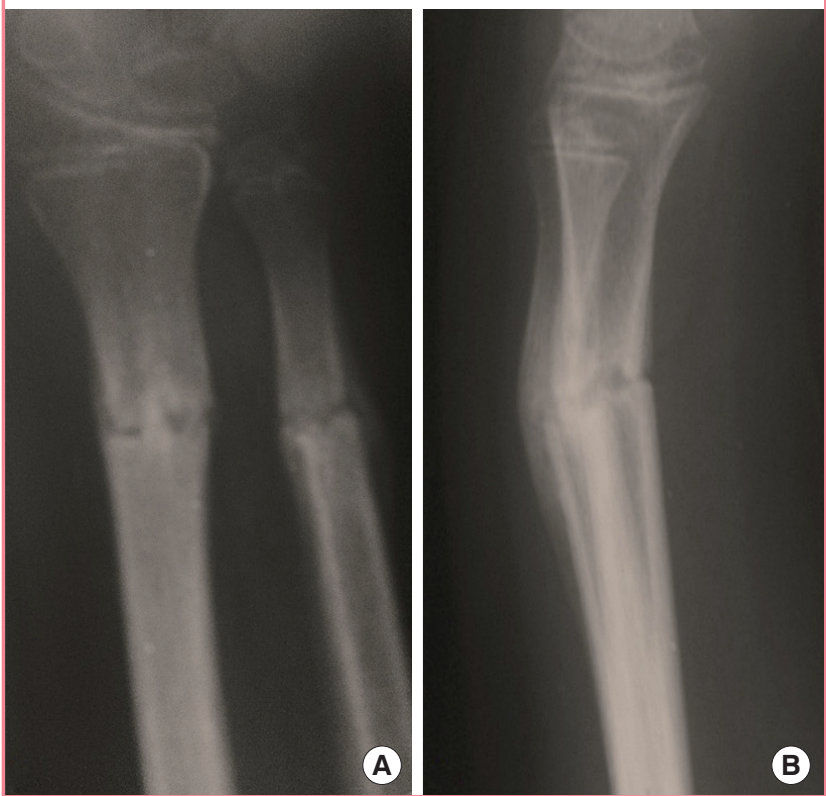

dle electromyography detected denervation of the abductor pollicis brevis. Radiographs showed that the fracture had healed with only a slight irregularity.

Eight months after the injury, surgical exploration of the median nerve was carried out. An anterior incision was performed

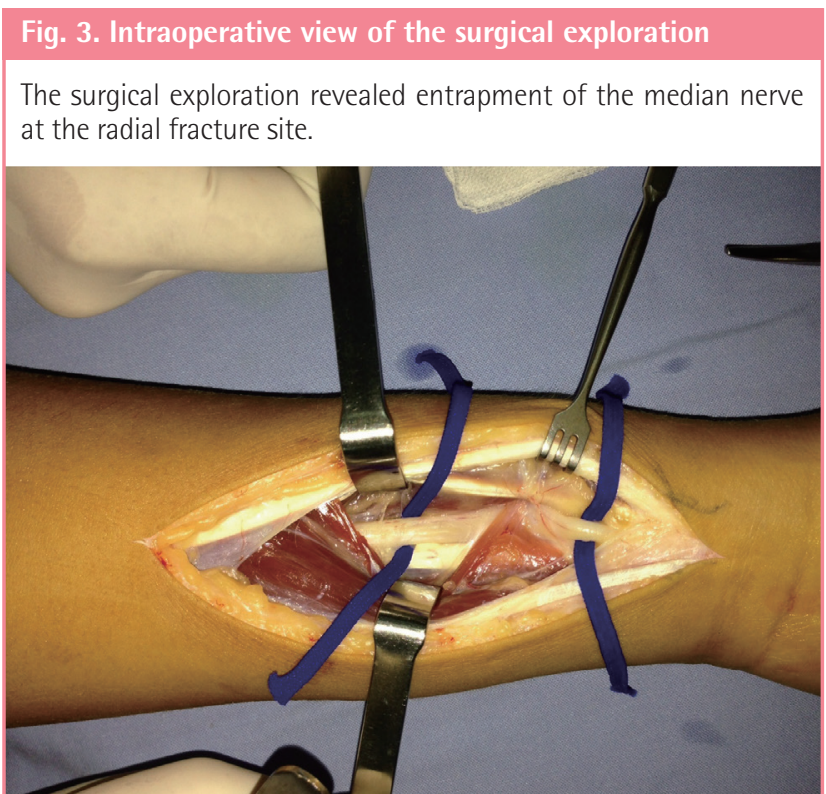

\section{Fig. 4. Intraoperative view after a neurolysis attempt \\ Neurolysis failed because the nerve was caught in the bony callus. Dissection was impossible. The picture shows a nerve discontinuity caused by attempting release, and a flattening in the distal part.}

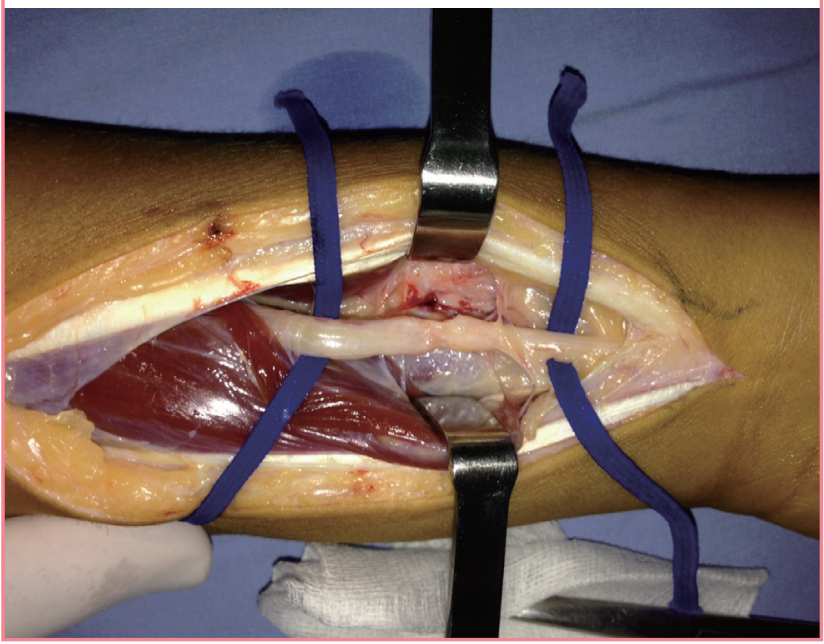

on the left forearm, centered on the area of the positive Tinel sign. The flexor muscles were split and the median nerve was identified. The nerve was trapped in the radius callus fracture (Fig. 3). Neurolysis was attempted, but was impossible (Fig. 4). After resection of the injured nerve and the neuroma, we found a loss of substance (Fig. 5). The possibility of a nerve suture without tension was tested with a nylon $9 / 0$ suture, but it was not possible, even with flexion of the wrist. A nerve graft was performed using the left sural nerve (Fig. 6).

Two years after surgery, follow-up revealed recovery of the abductor pollicis brevis, and 2-point discrimination was $6 \mathrm{~mm}$ on the thumbs and $10 \mathrm{~mm}$ on the index and the middle finger. 


\section{Fig. 5. Intraoperative view of nerve loss of substance}

The dissected part, the neuroma, and the glioma were resected, resulting into a loss of substance measuring $20 \mathrm{~mm}$.

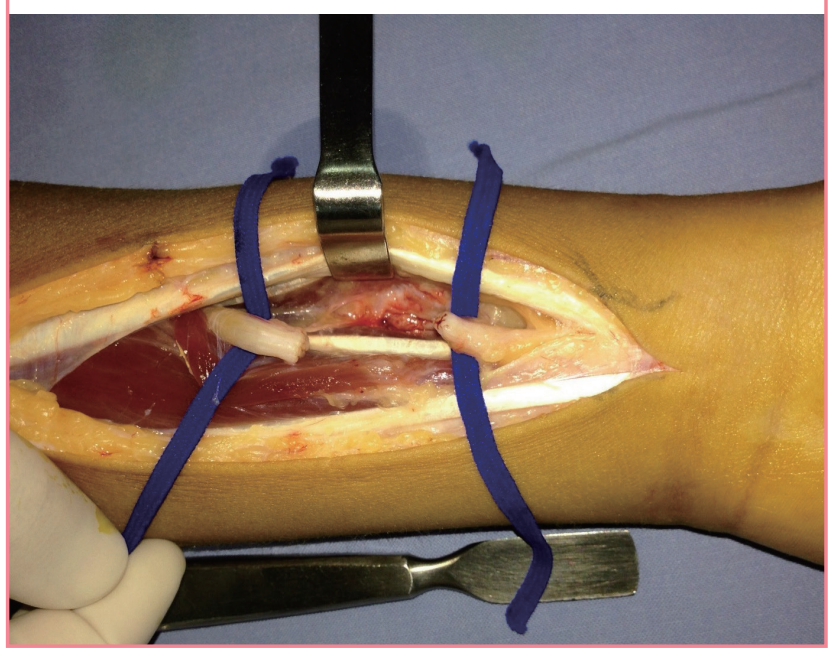

Fig. 6. Intraoperative view of the median nerve graft

After excision of the entrapped part, the median nerve defect was grafted using the sural nerve.

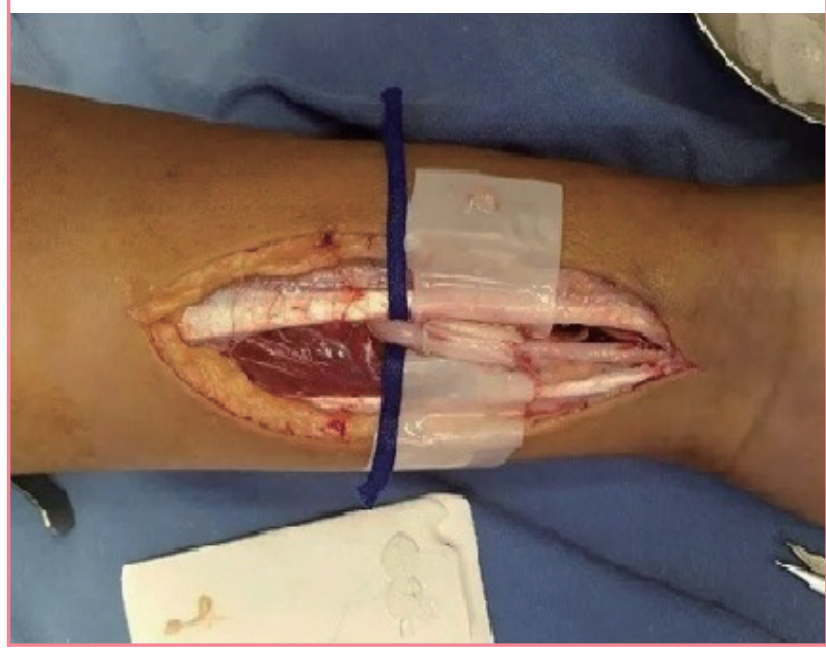

\section{DISCUSSION}

Median nerve entrapment in forearm fractures in children is uncommon. Eleven cases have reported in the literature from 1974 to 2016 (Table 1). The mean age of the affected patients was 11 years (range, 6-13 years). Except for the cases described by Nunley and Urbaniak [10] and Yeo et al. [3], in which the nerve entrapment was at an ulnar fracture site, the nine other cases were due to a radial fracture. The radial fractures were proximal in one case [7], at the mid-shaft in eight cases, and in the distal third in the two remaining cases and our case. Closed reduction was performed in eight cases $[1,3-8,11]$, an open reduction in one case [2], and no reduction was needed in two cases $[9,10]$.

\section{Fig. 7. The radiographic follow-up after 8 months}

$(A, B)$ The radiograph shows complete bone healing and bone remodeling. Note the bony canal (yellow arrow) at the healed radius fracture site.
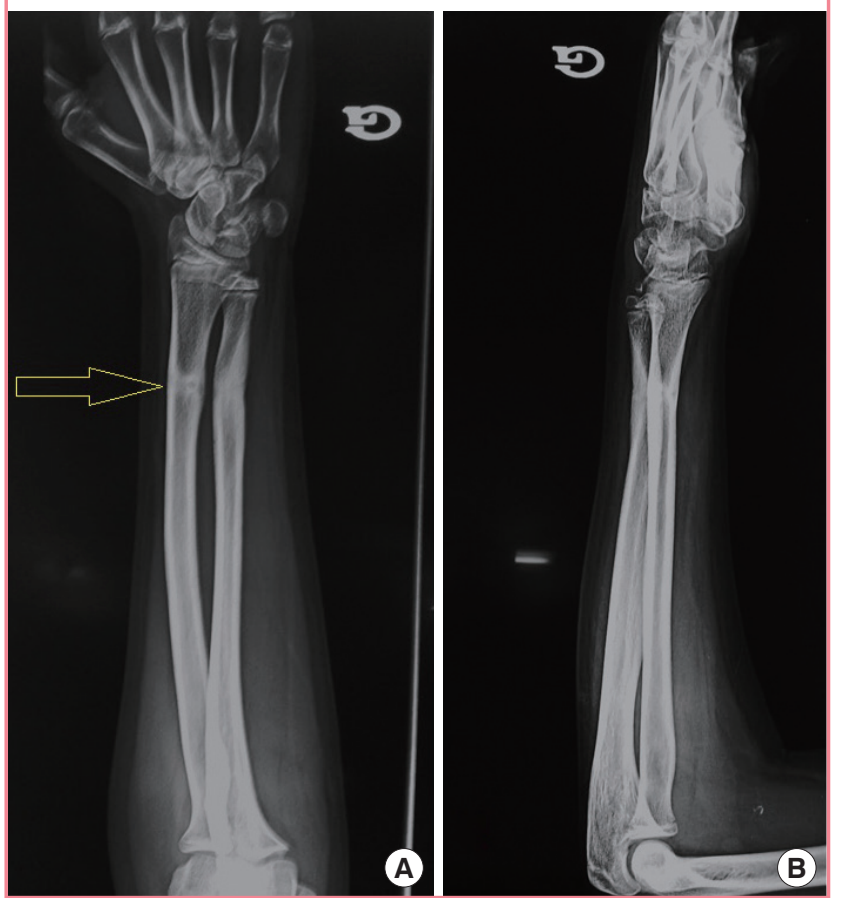

In addition to the median nerve, the interosseous nerve was involved in one case [8]. Except for the case described by Hurst and Aldridge [2], in which surgical exploration was immediately indicated, both the diagnosis and the surgical exploration were delayed. In the reported cases, the median nerve entrapment was released after 39 days to 24 months [7,11]. The reasons for the delayed diagnosis include the absence of clinician continuity in serial follow-up examinations, the unclear nature of complaints from affected children, and the assumption that such numbness will prove to be temporary.

The presence of a slight bony irregularity, a bony canal [10], or a bony spike at the site of the fracture may suggest median nerve entrapment [7]. However, those radiological irregularities are usually only appreciated postoperatively [7]. In our case, a bony canal was present on the anteroposterior view of the healed radial fracture (Fig. 7). Magnetic resonance imaging (MRI) was only performed in one case [3], although it is useful for tracing the median nerve course in the forearm. Yanagibayashi et al. [12] advocate the use of MRI earlier if entrapment is suspected. It enables immediate visualization of the entrapment, and surgical exploration can be promptly performed to release the entrapment.

Neurolysis and neurorrhaphy were the most common management procedures, and a median nerve graft was performed 


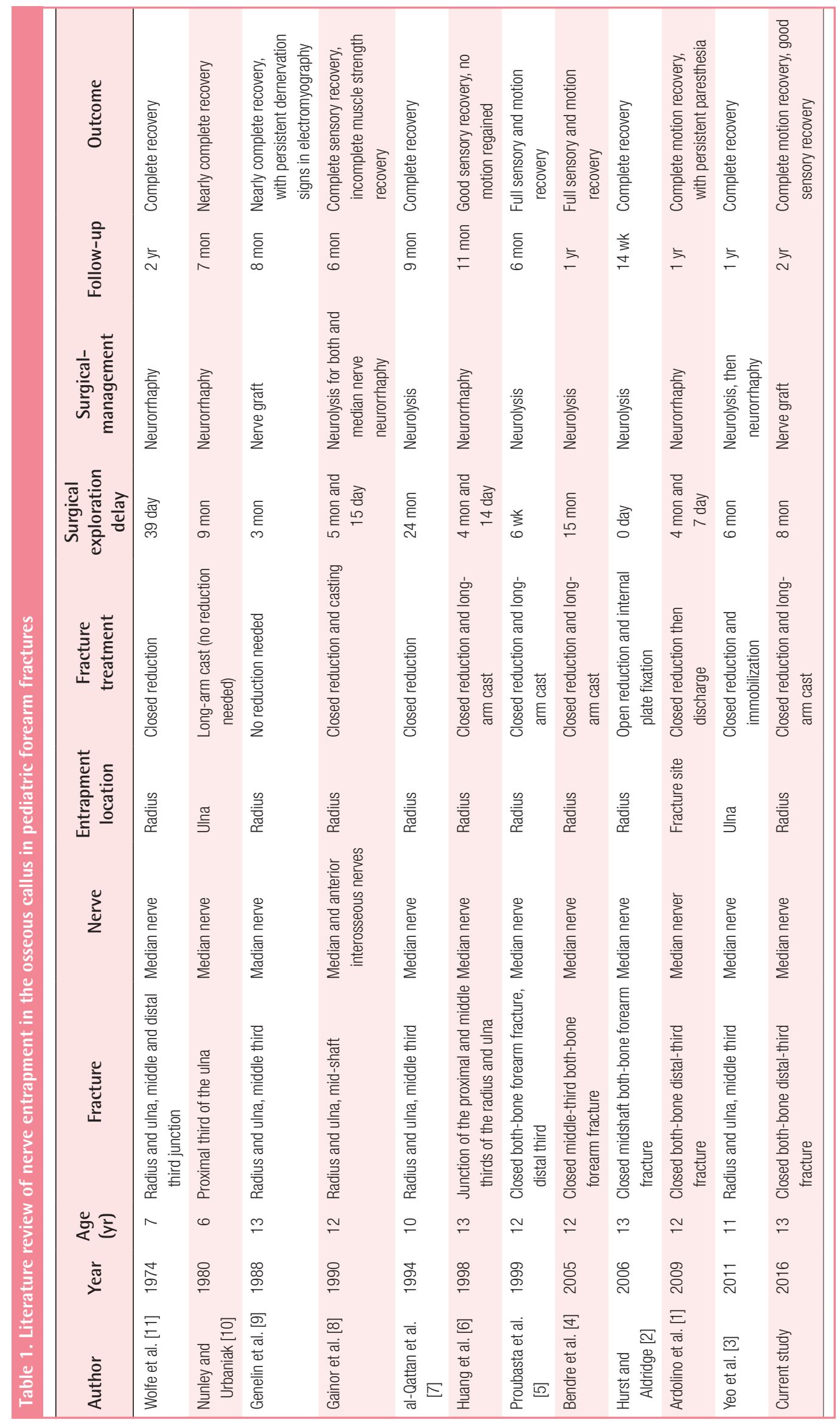


in one other case [9], as well as in our case. Fortunately, the literature has demonstrated that median nerve function shows excellent recovery in childhood, even with delayed surgery.

This case emphasizes the importance of a meticulous clinical examination before and after closed reduction to detect a nerve injury. We highlight the value of clinician continuity in serial follow-up examinations. Early exploration of persistent neurological deficits is advocated, and MRI may be useful.

\section{NOTES}

\section{Conflict of interest}

No potential conflict of interest relevant to this article was reported.

\section{Ethical approval}

The study was approved by the Habib Bourguiba University Hospital Ethics Committee (IRB No. 7-17) and performed in accordance with the principles of the Declaration of Helsinki. Written informed consents were obtained.

\section{Patient consent}

The patient provided written informed consent for the publication and the use of his images.

\section{Author contribution}

Clinical study, drafting, and approval of the manuscript: Fourati A. Literature review: Karra A. Critical revision: Ghorbel I. Electromyography study: Elleuch MH. Study supervision: Ennouri K.

\section{ORCID}

Amine Fourati https://orcid.org/0000-0003-3401-2010 Iyadh Ghorbel https://orcid.org/0000-0001-7395-4196

Amir Karra https://orcid.org/0000-0001-9827-2511

Mohamed Habib Elleuch https://orcid.org/0000-0002-74324839

Khalil Ennouri https://orcid.org/0000-0003-1387-1772

\section{REFERENCES}

1. Ardolino A, Webb D, Richards S. Median nerve entrapment in fracture callus following a paediatric forearm fracture: case report and review of the literature. Injury Extra 2009;40:274-6.

2. Hurst JM, Aldridge JM 3rd. Median nerve entrapment in a pediatric both-bone forearm fracture: recognition and management in the acute setting. J Surg Orthop Adv 2006;15: 214-6.

3. Yeo G, Prodger S, Latendresse K. Median nerve entrapment in a paediatric fracture of the ulna demonstrated by magnetic resonance imaging. J Hand Surg Eur Vol 2011;36:329-30.

4. Bendre A, Adeeb M, Malkan D. Median nerve entrapment in mid-shaft radius fracture callus. Eur J Trauma 2005;31: 407-8.

5. Proubasta IR, De Sena L, Caceres EP. Entrapment of the median nerve in a greenstick forearm fracture: a case report and review of the literature. Bull Hosp Jt Dis 1999;58:220-3.

6. Huang K, Pun WK, Coleman S. Entrapment and transection of the median nerve associated with greenstick fractures of the forearm: case report and review of the literature. J Trauma 1998;44:1101-2.

7. al-Qattan MM, Clarke HM, Zimmer P. Radiological signs of entrapment of the median nerve in forearm shaft fractures. J Hand Surg Br 1994; 19:713-9.

8. Gainor BJ, Olson S. Combined entrapment of the median and anterior interosseous nerves in a pediatric both-bone forearm fracture. J Orthop Trauma 1990;4:197-9.

9. Genelin F, Karlbauer AF, Gasperschitz F. Greenstick fracture of the forearm with median nerve entrapment. J Emerg Med 1988;6:381-5.

10. Nunley JA, Urbaniak JR. Partial bony entrapment of the median nerve in a greenstick fracture of the ulna. J Hand Surg Am 1980;5:557-9.

11. Wolfe JS, Eyring EJ. Median-nerve entrapment within a greenstick fracture: a case report. J Bone Joint Surg Am 1974; 56:1270-2.

12. Yanagibayashi S, Yamamoto N, Yoshida R, et al. Magnetic resonance imaging visualizes median nerve entrapment due to radius fracture and allows immediate surgical release. Case Rep Orthop 2015;2015:703790. 